\title{
The Challenge of Visualizing Patient Histories on a Mobile Device
}

\author{
Carmelo Ardito, Paolo Buono, and Maria Francesca Costabile \\ Dipartimento di Informatica, Università degli Studi di Bari, Italy \\ \{ardito, buono, costabile\}@di.uniba.it
}

\begin{abstract}
This paper presents a tool to display patient histories and to visually query patient data, stored in the hospital database, using a mobile device. Employing Information Visualization techniques, the developed tool is able to accommodate on the screen a good amount of information that physicians require in their analysis of the clinical cases. This work has been motivated by specific requests of physicians of a pediatric hospital treating children with neurological diseases.
\end{abstract}

\section{Introduction and Motivation}

Patient records are an important source of information for the physicians; they can be multifaceted and collected in time periods ranging from days to decades. For several diseases, physicians need to interpret rapidly a large number of clinical data collected during the patient disease history in order to formulate an accurate diagnosis and plan a treatment. Often a doctor needs to check such data out of the hospital, for instance when he is called on the phone for an emergency.

This paper presents a tool designed for a mobile device that displays he patient histories and permits to visually query patient data stored in the hospital database. The work is motivated by specific requests of physicians of a pediatric hospital treating children with neurological diseases. The challenge is to capture as much as possible information about the patient history on a limited display space, providing overview data as well as details. The employed visualization technique is inspired to Shneiderman's "information-visualization-seeking mantra": Overview first, zoom and filter, then details-on-demand [5. Providing data overview gives users indication of content and interconnections within an information domain. Zooming and filtering mechanisms allow users to concentrate on some portion of data, while mechanisms for showing details, when users request them, must also be included.

The work has been inspired from LifeLines, a general technique for visualizing summaries of personal histories [4. Displaying on a single screen the overview of multiple facets of records, this technique provides users with a better sense of type and volume of the available data. Other systems have been proposed to support medical personnel in their work. In particular, the systems presented in [1] and [3] use handhelds PC connected, via Wireless Networks, to centralized databases to allow doctors and nurses to check patient clinical records. However, these works do not provide details about user interface features. 
The development of the mobile application we describe in this paper is carried out in collaboration with physicians of "Giovanni XXIII" pediatric hospital in Bari, Italy. A field study with contextual interviews has been performed during the requirements analysis to better understand the medical domain, how the physicians operate and the main features of the application to be implemented. User observation methods and principles of participatory design have proven to be effective in user interface design. To our knowledge, our application is the first one adapting LifeLines on a PDA, thus visualizing on a small screen as much as possible patient data useful to the physicians in their analysis of the clinical cases. More details on the field study and on the requirement analysis can be found in 22. In the remainder of this paper, we will briefly describe the visualization technique to display patient histories on a PDA.

\section{PHiP: Patient History in Pocket}

PHiP is a tool designed to support the neurologists in the treatment of patients with epilepsy; it is intended to make available on mobile devices some sections of patient records and to implement features and functionality that neurologists consider needful to treat such neurological diseases. Several important indications emerged from the field study performed during the requirements analysis. First of all, it is very important for neurologists to analyze the patient history and have information such as periods during which the patient was hospitalized, frequency of seizures, different combinations of drugs the patient took during his/her disease history. Another indication is that the drugs that really work for children affected by epilepsy are a small number, less than twelve; some combinations of them are prescribed to the patients during periods of time and neurologists must know what was prescribed. We therefore realized that neurologists could greatly benefit from having an overview of multi-dimensional, time-oriented data related to all patient hospitalizations, medical controls, therapies. After designing and evaluating with users several prototypes, the main screen of the current PHiP interface is shown in Figure 1, This screen is displayed once the user (we suppose a male neurologist) has specified the name of the patient and the history period he wants to see: the default is the last five years (or the whole history if it covers less than five years). The name of the patient is shown at the very top of the screen. The display is divided in two main areas: the bottom one, below the green bar with 6/1996 and 1/2001 at the sides, is the overview area of the patient history; the top larger area is the focus area and shows a zoom in a five months time period.

Let us first concentrate on the bottom area that, in Figure 1, indicates that the overview spans from August 1996 to December 2000. The user can change the range of the displayed time period by clicking on the green bar and selecting a value from a combo box that will appear. In this overview area, various information is summarized through colored line segments that we call bars. The bars along a line immediately below the ticker green bar represent hospitalizations or medical controls, in red and green respectively. The bars on the lines below 
represent the drugs prescribed in the shown time period. The white strip in the overview area represents a lens that permits to zoom on the information covered by the lens, which spans over five months, and shows it in the focus area. The user can quickly move the lens by clicking on another point in the overview area, or gradually move it by clicking on the single-arrow buttons at the extremes of the overview area. The slide bar at the left of the overview area permits to modify the thickness of the colored bars choosing among three different sizes: this because interacting with previous prototypes, some doctors said that it was difficult to distinguish the bars. This overview area can be eliminated by simply clicking on the button above this slide bar. In this way the focus area will be enlarged. Another click will show the overview again.

Detailed information is displayed in the focus area. As for the overview area, the user can change the range of the displayed interval by clicking on the green bar and selecting a value from a combo box. In this way, the user can visualize even a short interval if he wants more details in that time interval. The lens in the overview area is updated accordingly. Hospitalizations and medical controls are shown along the top line below the month bar using red and green colors: they never overlap because a patient might either be hospitalized or go to the outpatient clinic for a medical control. To help the user recall that red indicates hospitalizations, the icon representing a bed at the left of the line is in red, while med-

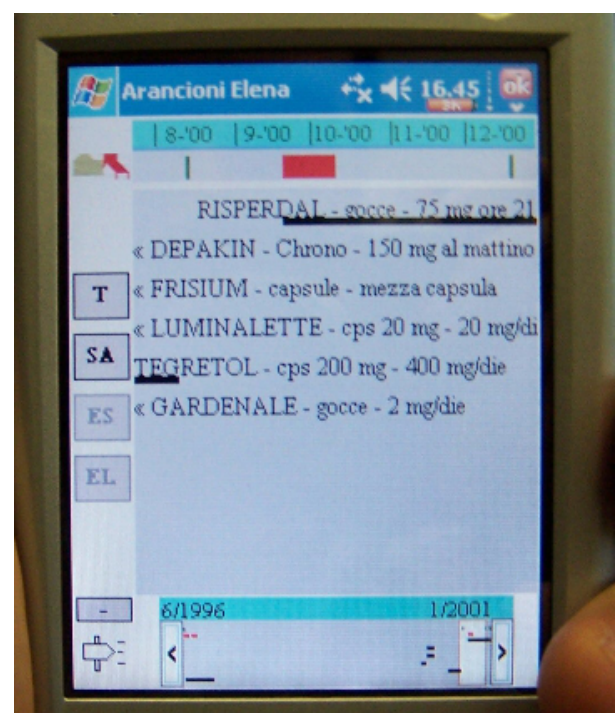

Fig. 1. An example of PHiP interface ical controls are in green, which is the same color of the folder icon at the left of the bed icon. In Figure 1, in the period August-December 2000 the patient had two medical controls, one in August and one in December, and was hospitalized from end of September to beginning of October.

In the remaining part of the focus area, details about prescribed drugs are shown. Each drug is indicated by a label that explains the name and the posology. If a drug was prescribed in the displayed period, a black bar starts from the day in which the patient took it and ends when he/she stopped the treatment. For example, Figure 1 shows that from the second half of September, the drug RISPERDAL is taken daily in a quantity of 75 milligrams at $9 \mathrm{pm}$. If a drug was prescribed in a period that is not displayed, the label is shown without the bar and an arrow indicates if it was taken in a previous or in a following period. In Figure 1 DEPAKIN is a drug that was taken before August 2000. Two arrows, one at left and one at right, are shown if the drug was taken in a previous period 
as well as in a following one. This because for the neurologist it is important to know if a patient already got a drug during a certain time of his/her life.

In Figure 1, two buttons on the left of the focus area are active: one has the $T$ label, since the focus area shows the patient therapy in the selected time interval. The button with $S A$ label allows the user to display patient personal data. The other two buttons become active if the user requires detailed information. More specifically, a click on a period in which the patient was hospitalized, or on a medical control, allows the physician to see details related to that event. This click has several effects. First of all, the drugs prescribed in the clicked period change color and become blue. The ES an EL buttons on the left of the focus area are activated. By clicking on $E L$, the list of laboratory tests referring to the selected hospitalization or medical control appears in the focus area, replacing the previously visualized data. The user can also query such data to see more details on the tests. Similarly, by clicking on ES, the physician obtains the list of instrumental tests. Such detailed views can be seen in [2].

\section{Conclusion}

The aim of PHiP, Patient History in Pocket, is to increase the quality of neurological diseases treatment, by supporting physicians with a tailored tool.

The field study carried out in a hospital revealed that the primary need for neurologists is the availability, in different contexts, of patient histories. Thus, a mobile device is suitable to query patient data stored in the hospital database. The main challenge was to accommodate the patient data, on the small screen of the PDA, providing an overview of the most significant data and their temporal sequence. Prototype testing and interviews with neurologists are confirming that the developed application satisfies their needs. They appreciate very much that the tool is useful, easy to understand and use much more than they expected.

\section{References}

1. M. Ancona, G. Dodero, V. Gianuzzi, F. Minuto, and M. Guida. Mobile computing in a hospital: the WARD-IN-HAND project. In Proceedings of the 2000 ACM Symposium on Applied Computing, SAC 2000, pages 554-556, 2000.

2. C. Ardito, P. Buono, and M. F. Costabile. Visualizing patient histories on mobile devices. Technical Report TR 05-03-01, LACAM - IVU group, Department of Computer Science, University of Bari, Italy, March 2005.

3. J. Bardram, T. A. Kjaer, and C. Nielsen. Supporting local mobility in healthcare by application roaming among heterogeneous devices. volume 2795 of Lecture Notes in Computer Science, pages 161-176. Springer-Verlag, 2003.

4. C. Plaisant, B. Milash, A. Rose, S. Widoff, and B. Shneiderman. Lifelines: Visualizing personal histories. In Proceedings of the ACM Conference on Human Factors in Computing Systems, CHI-1996, pages 221-227. ACM Press, 1996.

5. B. Shneiderman and C. Plaisant. Designing user interfaces. Addison Wesley, Washington, D.C., 2004. 